\title{
Audit of adult inpatients receiving parenteral nutrition - 3-year trends since setting up a nutrition support team
}

\author{
L. M. Gregory, M. Bennett-Marsden and T. M. Trebble \\ Queen Alexandra Hospital, Portsmouth PO6 3LY, UK
}

Parenteral nutrition is a well-recognised method of providing nutrition to patients with short- or long-term intestinal failure and is best managed by a coordinated team approach ${ }^{(1-3)}$. In Portsmouth, the nutrition support team (NST) has been operating for 6 years. Twice weekly ward rounds, daily reviews by members of the NST, regular teaching and support to nursing and medical staff, have made a positive contribution to the management of these patients. Eight standards in the local policy for the management of adult patients on parenteral nutrition are audited annually, based on good practice ${ }^{(2,3)}$. An electronic database has been designed and audit data collected by members of the team since 2007 - results are presented and trends over the last 3 financial years explored.

\begin{tabular}{cccc}
\hline & Patients receiving PN $(n)$ & Duration of PN episodes $(d)$ & Median duration of PN episode $(d)$ \\
\hline $2007-2008$ & 82 & $1-62$ & 6 \\
$2008-2009$ & 81 & $1-111$ & 6 \\
$2009-2010$ & 71 & $2-40$ & 7 \\
\hline
\end{tabular}

The drop in patients receiving PN is $13.5 \%$. The median duration compares with the $8 \mathrm{~d}$ from the National Confidential Enquiry into Patient Outcome and Death (NCEPOD) ${ }^{(1)}$. The percentage of patients and the duration of PN delivery are given below:

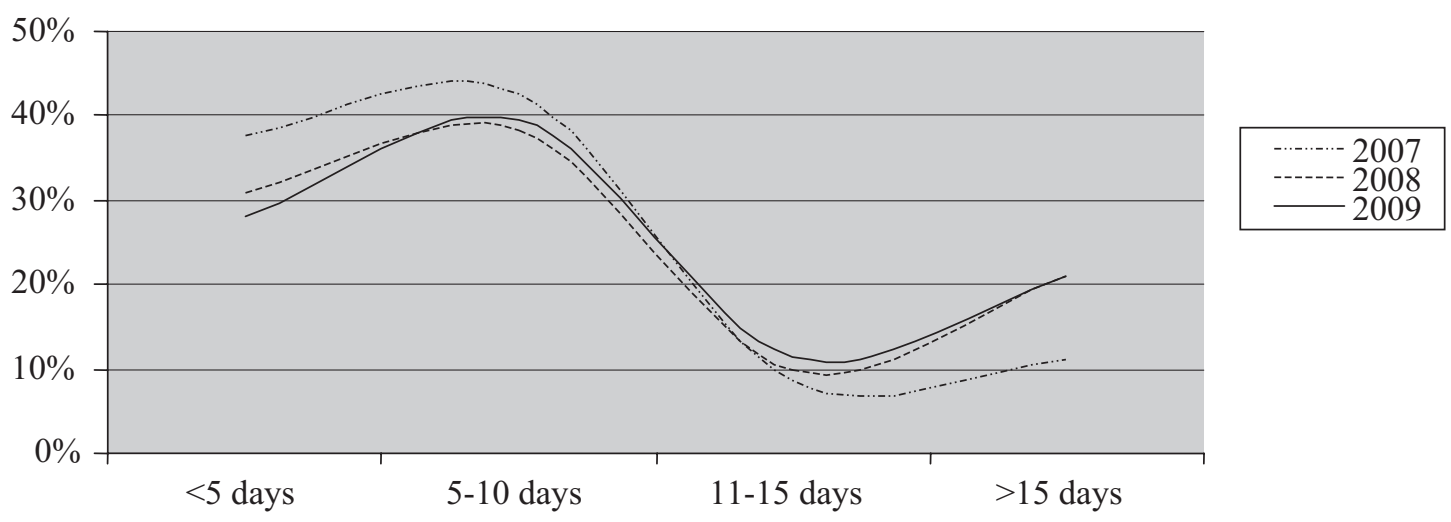

The number of different clinical specialties requesting PN has reduced over the last 3 years, especially those episodes of short duration ( $3 \mathrm{~d}$ or less), possibly reflecting a greater control on inappropriate usage. The proportion of short-duration PN from the NCEPOD results ${ }^{(1)}$ recently published was $20 \%$, compared to 18,10 and $20 \%$ in Portsmouth, over each of the 3 years. The percentage of patients receiving PN for $3 \mathrm{~d}$ or less by specialty was:

\begin{tabular}{lccc}
\hline & $2007-2008$ & $2008-2009$ & $2009-2010$ \\
\hline Colorectal surgery & $34 \%$ & $50 \%$ & $50 \%$ \\
Upper GI surgery & $26 \%$ & $25 \%$ & $29 \%$ \\
Gastroenterology & $7 \%$ & $0 \%$ & $0 \%$ \\
Other & $33 \%$ & $25 \%$ & $21 \%$ \\
\hline
\end{tabular}

The proportion of PN for patients from specialties other than GI surgery has decreased over the 3 years. The most common reason for PN was postoperative ileus which increased from $31 \%$ to $50 \%$ over the 3 years, the duration of which is difficult to predict. These trends may reflect a decrease in inappropriate $\mathrm{PN}^{(1)}$. The number of patients deemed at risk of re-feeding syndrome following local guidelines increased in the last year.

\begin{tabular}{cccc}
\hline & At risk $(n)$ & Not at risk $(n)$ & Unknown $(n)$ \\
\hline $2007-2008$ & 54 & 34 & 12 \\
$2008-2009$ & 52 & 34 & 14 \\
$2009-2010$ & 63 & 30 & 7 \\
\hline
\end{tabular}

A rise in the last year reflects the findings of $\mathrm{NCEPOD}^{(1)}$ who deemed $60.3 \%$ were at risk compared to $49.8 \%$ as documented. The percentage of referrals, where adequate biochemistry was available for the nutritional assessment to be undertaken, was:

\begin{tabular}{ll}
\hline $2007-2008$ & $58 \%$ \\
$2008-2009$ & $85 \%$ \\
$2009-2010$ & $78 \%$ \\
\hline
\end{tabular}

Incomplete biochemistry on referral will potentially compromise the appropriateness of the initial prescription ${ }^{(1-3)}$. These data support the effectiveness of a multidisciplinary nutrition team in managing PN within a district general hospital.

1. Stewart JAD, Mason DG, Smith N et al. (2010) A Mixed Bag: An Enquiry into the Care of Hospital Patients Receiving Parenteral Nutrition. London: National Confidential Enquiry into Patient Outcome and Death.

2. National Institute for Health and Clinical Excellence (2006) Nutrition Support in Adults: Oral Nutrition Support, Enteral Tube Feeding, and Parenteral Nutrition. Clinical Guideline 32, London: National Institute for Health and Clinical Excellence.

3. ASPEN Board of Directors (2002) Guidelines for the use of parenteral and enteral nutrition in adult and pediatric patients. JPEN J Parenter Enteral Nutr 26, Suppl 1, 1SA-138SA. 